\title{
Wettbewerb im Deutschlandtakt
}

\begin{abstract}
Die neue Bundesregierung hält an dem verkehrspolitischen Ziel einer Steigerung des Verkehrs auf der Schiene fest. Dazu sieht sie im Koalitionsvertrag neben einer weiteren Erhöhung der Investitionen in die Schieneninfrastruktur auch eine prioritäre Ausrichtung der Finanzierung auf den Deutschlandtakt vor. Damit durch die angestrebte Umgestaltung tatsächlich eine Verkehrsverlagerung auf die Schiene und eine Steigerung des Schienenverkehrs erreicht werden kann, sollte frühzeitig auf eine wettbewerbliche Ausgestaltung geachtet werden. Dafür sind regulatorische Anpassungen notwendig. Ein geeignetes Instrument ist die Umgestaltung des Schienenpersonenfernverkehrs hin zu einem Ausschreibungs- bzw. Konzessionsmodell. Hierdurch könnte die Bedienung der vordefinierten Deutschlandtakt-Strecken sichergestellt werden. Die Umgestaltung kann zudem zu einer Steigerung des Wettbewerbs, einem attraktiveren Bahnmarkt und somit auch zu einer Verkehrssteigerung auf der Schiene beitragen.
\end{abstract}

\begin{abstract}
Der Koalitionsvertrag der neuen Bundesregierung sieht ein Festhalten an den Zielen der Verkehrssteigerung auf der Schiene vor. So soll, wie bereits im Koalitionsvertrag 2018 angestrebt, eine Steigerung des Schienengüterverkehrs im intermodalen Vergleich auf $25 \%$ bis 2030 sowie eine Verdoppelung der Verkehrsleistung im Schienenpersonenverkehr erreicht werden. Ein Mittel, das hierfür zum Einsatz kommen soll, ist der Deutschlandtakt. Dabei handelt es sich um einen Paradigmenwechsel für den deutschen Schienenverkehr: Bislang wurde der Fahrplan basierend auf dem bestehenden Schienennetz erarbeitet. Nun soll jedoch - umgekehrt - der Infrastrukturausbau anhand eines vorkonzipierten Zielfahrplans erfolgen. Ein solcher Fahrplan für den Deutschlandtakt wird bereits

(C) Der/die Autor:in 2022. Open Access: Dieser Artikel wird unter der Creative Commons Namensnennung 4.0 International Lizenz veröffentlicht (creativecommons.org/licenses/by/4.0/deed.de).

Open Access wird durch die ZBW - Leibniz-Informationszentrum Wirtschaft gefördert.

* Der Beitrag geht im Wesentlichen zurück auf das 8. Sektorgutachten Bahn „Wettbewerb in den Takt!“ der Monopolkommission (2021).
\end{abstract}

Prof. Dr. Jürgen Kühling, LL. M., ist Vorsitzender der Monopolkommission und Inhaber des Lehrstuhls für Öffentliches Recht, Immobilienrecht, Infrastrukturrecht und Informationsrecht an der Universität Regensburg.

Dr. Katja Greer ist Senior Analystin bei der Monopolkommission in Bonn. seit einigen Jahren unter Einbeziehung der Marktteilnehmenden erarbeitet. Durch das gezielte Abstimmen von Verkehrsleistungen könnten im Vergleich zum Status quo mehr Verkehre über das bestehende bzw. anzupassende Schienennetz geleitet werden. Zudem würden diese Kapazitäten durch ein konsequentes Abstimmen und unter Berücksichtigung der Nachfrage geschickter dem Schienenpersonennahverkehr (SPNV), dem Schienenpersonenfernverkehr (SPFV) sowie dem Schienengüterverkehr (SGV) zugeordnet werden.

Die Einführung dieses bundesweit geltenden integralen Taktfahrplans dürfte für Reisende wie auch für Eisenbahnverkehrsunternehmen Vorteile mit sich bringen. Durch die Vorgabe regelmäßig wiederholender Fahrangebote, z. B. im Halbstundentakt, und durch das Abstimmen der Fahrten über gemeinsame Bahnhofshalte sollten sich aus Sicht der Reisenden kürzere Fahrzeiten und Wartebzw. Umsteigezeiten ergeben. Aus Sicht von Güterverkehrsunternehmen könnten ebenso Vorteile geschaffen werden, da durch das Abstimmen des Fahrplans Freiräume für Güterverkehrsleistungen eingeplant werden können. So kann der Deutschlandtakt den Schienenverkehr attraktiver gestalten und zu dem gewünschten Ziel „Mehr Verkehr auf die Schiene“ beitragen.

Voraussetzung ist eine funktionsfähige Infrastruktur

Die infrastrukturellen Vorbereitungen für den Deutschlandtakt haben vor allem in den Jahren 2020/2021 an Fahrt aufgenommen. So wurde im Juli 2020 der dritte und letzte Gutachterentwurf zum Zielfahrplan wie auch der Masterplan Schienenverkehr veröffentlicht (BMVI, 2020a; BMVI, 2020b). Letzterer erörtert neben weiteren Maßnah- 
men für einen „starken“ Schienenverkehr den besonderen Handlungsbedarf hinsichtlich des Deutschlandtakts und benennt wichtige Neu- und Ausbaustrecken. Im August 2021 folgten der Abschlussbericht zum Zielfahrplan Deutschlandtakt sowie eine gesamtwirtschaftliche Bewertung des Gesamtplanfalls Deutschlandtakt (BMVI, 2021). Durch diese rückten die notwendigen Infrastrukturmaßnahmen in den vordringlichen Bedarf des Bundesverkehrswegeplans 2030 auf und sollen in den kommenden Jahren geplant und umgesetzt werden.

Damit diese Umsetzung gelingen kann, sind höhere Investitionen in die Infrastruktur notwendig. Dies zeichnet sich speziell beim Vergleich der erwarteten Ausgaben mit den bisherigen Finanzierungsmaßnahmen ab. Für den Neu- und Ausbau der priorisierten DeutschlandtaktMaßnahmen sind laut dem Bundesministerium für Verkehr und digitale Infrastruktur (BMVI) rund $42 \mathrm{Mrd}$. Euro einzuplanen. Mit Berücksichtigung weiterer notwendiger Maßnahmen sowie der Digitalisierung werden die Infrastrukturkosten des Vorhabens Deutschlandtakt sogar auf mehr als 100 Mrd. Euro geschätzt (Böttger, 2020). Bisher stehen für diese Baumaßnahmen im Bundeshaushalt rund 1,5 Mrd. Euro pro Jahr zur Verfügung. Ab 2023 dürfte sich das Volumen auf 2 Mrd. Euro pro Jahr erhöhen. Doch auch mit dieser relativ geringen Erhöhung zeichnet sich aktuell eine Unterfinanzierung der Infrastrukturvorhaben ab. Diese dürfte den Schienenausbau verzögern und könnte die Fertigstellung der geplanten Infrastruktur für den Deutschlandtakt um Jahrzehnte hinausschieben. Um mit dem Deutschlandtakt in den kommenden Jahren eine Verkehrssteigerung auf der Schiene zu erzielen, sind die finanziellen Mittel daher drastisch zu erhöhen.

\section{Kein integraler Taktfahrplan ohne Pünktlichkeit}

Eine weitere Voraussetzung, die vor allem im Fall einer Umsetzung der vordefinierten Trassen notwendig wird, ist die Pünktlichkeit. Nur wenn die Verkehrsleistungen pünktlich erfolgen, können die Vorteile einer aufeinander abgestimmten Planung realisiert werden. Die Pünktlichkeit im deutschen Schienenverkehr lässt jedoch weiterhin zu wünschen übrig. Vor der Corona-Pandemie litt insbesondere der SPFV unter Verspätungen. So waren 2019 ausschließlich 75,9\% aller Fernverkehrszüge der Deutschen Bahn AG pünktlich. ${ }^{1}$ Unter den besonderen Bedingungen des Jahres 2020 mit eingeschränkter Mobilität und Aufrechterhaltung des Fernverkehrs waren es $81,8 \%$. Dabei haben Verspätungen unterschiedliche Ursachen. Etwa ein Drittel der Verspätungen geht auf die Eisenbahnverkehrsunternehmen zurück - ein mindestens ebenso hoher Anteil auf den Infrastrukturbetreiber.

1 Dabei gilt ein Personenzug als pünktlich, wenn die planmäßige Ankunftszeit um weniger als sechs Minuten überschritten wurde.
Um die Pünktlichkeit für den Deutschlandtakt zu verbessern, könnten insbesondere Anreizmechanismen für den Infrastrukturbetreiber und eindeutige Haftungsregelungen helfen, wie sie die Monopolkommission (2019) empfiehlt. Des Weiteren dürfte eine Ausweitung des rollenden Materials die Pünktlichkeit steigern. Durch das Vorhalten von Reservezügen an gewissen Hauptbahnhöfen könnten diese im Falle unpünktlicher Züge notfalls an den jeweiligen Bahnhöfen die Anschlussverbindungen aufrechterhalten. Hierdurch dürften weitere Investitionen notwendig werden.

Wettbewerb fördert Zielsetzung des Deutschlandtakts

Ausgehend davon, dass die Schieneninfrastruktur entsprechend dem Zielfahrplan ausgebaut wird, stellt sich die Frage, wie und unter welchen Bedingungen die Bedienung der Infrastruktur erfolgen soll. Die Monopolkommission weist in ihrem aktuellen, achten Sektorgutachten Bahn darauf hin, dass möglichst frühzeitig auf eine wettbewerbliche Ausgestaltung des Deutschlandtakts hingearbeitet werden sollte (Monopolkommission, 2021). Grund hierfür sind die Vorteile, die ein fairer Wettbewerb im Bahnmarkt mit sich bringt. So fördert die wettbewerbliche Ausgestaltung eine möglichst - qualitativ und preislich - gute Verkehrsbedienung und volkswirtschaftlich sinnvolle Aufgabenteilung. ${ }^{2}$ Der intramodale Wettbewerb, d.h. der Wettbewerb durch weitere Eisenbahnverkehrsunternehmen, wie auch der intermodale Wettbewerb, d.h. im Vergleich zu weiteren Verkehrsträgern wie dem motorisierten Individualverkehr, führen zu besseren Angeboten. Dies verdeutlichte die Fernbusliberalisierung, die günstigere Tickets und die Einführung von WLAN in Zügen förderte. Eine wettbewerbliche Ausgestaltung des Deutschlandtakts kann zu dem Ziel, den Schienenverkehr zu steigern, beitragen, da sie die Attraktivität des Bahnmarkts erhöht. Daher sollten möglichst faire und wettbewerblich sinnvolle Bedingungen für die Umsetzung des Deutschlandtakts geschaffen werden, damit die Vorgabe eines integralen Taktfahrplans nicht dazu führt, die marktmächtigen Verkehrsunternehmen zu stärken und Konkurrenz zu verhindern.

\section{Notwendigkeit eisenbahnrechtlicher Anpassungen}

Offen ist derzeit noch, wie die Eisenbahnverkehrsunternehmen im SPFV, SPNV und SGV die Leistungen des Deutschlandtakts erbringen werden. Grundsätzlich wäre es möglich, dass der Infrastrukturausbau auf Grundlage des Zielfahrplans umgesetzt wird, ohne dass rechtliche Anpassungen für die Erbringung von Eisenbahnverkehrsleistungen angestrebt werden. In diesem Fall stünde den Eisenbahnverkehrsunternehmen nach einer umfängli-

2 Zieldefinition § 1 Abs. 5 Allgemeines Eisenbahngesetz. 
chen Bauphase ein besseres bzw. umfänglicheres Infrastrukturnetz zur Verfügung, das sie für die Erbringung der Verkehrsleistungen nutzen könnten. Allein durch das Hinzukommen weiterer Strecken dürfte sich bereits eine Verkehrssteigerung ergeben. Allerdings wird gerade aus dem Abstimmen der Strecken und der Bahnhofshalte ein Mehrwert für Reisende durch verkürzte Fahr- und Umsteigezeiten erwartet. Daher ist zu hinterfragen, ob sich ohne eisenbahnrechtliche Anpassungen ein Fahrplan mit entsprechend abgestimmten Fahrten für den SPNV und SPFV ergeben könnte.

Im SPNV erscheint eine Abstimmung des Fahrplans langfristig sehr realistisch. Der bestehende regulatorische Rahmen im SPNV sieht einen Ausschreibungswettbewerb vor. Aufgabenträger definieren die regional zu erbringenden Leistungen bzw. regionale Netze und schreiben diese für mehrere Jahre aus. Eisenbahnverkehrsunternehmen bewerben sich und bedienen diese im Fall eines Zuschlags gemäß den im Verkehrsvertrag formulierten Bedingungen. Der Ausschreibungswettbewerb im SPNV steht einer Einführung des Deutschlandtakts daher grundsätzlich nicht entgegen, sofern Aufgabenträger die auszuschreibenden Netze an den Deutschlandtakt anpassen. Dies zeigt sich auch daran, dass sich in einigen Bundesländern der Regionalverkehr an integralen Taktfahrplänen ausgerichtet hat. So gibt es beispielsweise den Baden-Württemberg-Takt und integrale Taktfahrpläne in Rheinland-Pfalz oder auch in Nordrhein-Westfalen.

Ein Problem könnte allerdings der freie Marktzugang im SPFV sein. Im SPFV wie auch im SGV steht der Marktzugang entsprechend dem Open-Access-Modell allen Eisenbahnverkehrsunternehmen offen. Über Streckenführungen, Wiederholungen von Fahrten, Tarife und die weitere Ausgestaltung, wie beispielsweise Bahnhofshalte, entscheiden Eisenbahnverkehrsunternehmen im SPFV grundsätzlich selbst. Daher ist gerade beim Fernverkehr damit zu rechnen, dass sich Eisenbahnverkehrsunternehmen nicht an vordefinierte Strecken und Zeiten halten. Ganz im Gegenteil dürften Eisenbahnverkehrsunternehmen im SPFV die bestehende Infrastruktur immer dazu nutzen, eigenwirtschaftliche Strecken anzumelden und zu bedienen. Ohne eine Anpassung des rechtlichen Rahmens im SPFV besteht die Gefahr, dass nicht alle vorkonstruierten Trassen des Deutschlandtakts im Fernverkehr bedient werden, da sie nicht zwingend eigenwirtschaftlich sind.

Auswirkungen hat diese Problematik auch auf den SGV. Sollte der Fahrplan im SPFV stark von den vordefinierten Strecken des Deutschlandtakts abweichen, dürften die im SGV erwarteten Fahrzeitverkürzungen, die mit der abgestimmten Kapazitätsplanung im Deutschlandtakt einhergehen, ausbleiben. Ohne rechtliche Anpassung dürfte es kaum möglich sein, das Problem der Kapazitätszuordnung und effizienten Kapazitätsaufteilung zur Steigerung der Schienenverkehrsleistungen zu lösen. Somit ist es wenig wahrscheinlich, dass sich im gegenwärtigen Marktmodell allein durch die Infrastrukturplanung zum Deutschlandtakt eine Verbesserung für den SGV einstellt.

\section{Erprobung des Deutschlandtakts}

Als Reaktion auf den eisenbahnrechtlichen Anpassungsbedarf reagierte die Gesetzgebung im Juni 2021 bei der Weiterentwicklung des Eisenbahnregulierungsgesetzes (ERegG). Ein wesentlicher Bestandteil der Gesetzesänderung ist die Einführung des $\S 52 a$ ERegG. Hierbei handelt es sich um eine Erprobungsklausel, mit der Erfahrungen über neue Modelle der Kapazitätsnutzung und der Fahrplanerstellung gesammelt werden sollen. Im Grunde ist das Vorgehen klar strukturiert: Das BMVI wählt Pilotprojekte aus. Als Betreiber der Schienenwege stellt die DB Netz AG für die jeweiligen Projekte je ein neues Kapazitätsnutzungsmodell auf, das nach Absprache mit den Marktteilnehmenden daraufhin erprobt wird.

Im Vordergrund der Erprobung steht die Kapazitätszuweisung. Diese ist im Schienenverkehr mit den Marktmodellen im SPFV und SGV und dem einhergehenden freien Marktzugang bisher nicht rechtlich vorgesehen. Somit bildet die Erprobungsklausel eine Ausnahme von den bestehenden regulatorischen Marktvorgaben und eine Alternative zum bestehenden Vergabesystem. Dabei sieht die Erprobungsklausel zudem vor, dass die Festlegung der Konstruktionsparameter, anhand derer das zu erprobende Modell erstellt wird, „einem wirksamen Wettbewerb nicht entgegenstehen" darf. ${ }^{3}$ Sofern die Eisenbahnverkehrsunternehmen die von der DB Netz AG vorgeplanten Kapazitäten anmelden und sich an die jeweiligen Bedingungen halten, könnte dieses Verfahren über den Kapazitätsnutzungsplan, flankiert von weiteren rechtlichen Anpassungen, zu einer besseren Kapazitätsnutzung verhelfen. Kapazitätsnutzungspläne und langfristige Kapazitätsnutzungskonzepte werden z. B. auch in der Schweiz angewendet.

\section{Weitere rechtliche Anpassungen}

Die Erprobungsklausel stellt eine erste und durchaus sinnvolle Möglichkeit dar, einzelne Abschnitte des Deutschlandtakts umzusetzen. Es bleibt abzuwarten, wie die Beteiligung der Marktteilnehmenden verlaufen wird und wie sich die ersten Projekte entwickeln werden. Für eine bundesweite Einführung des Deutschlandtakts sind allerdings weitere rechtliche Anpassungen notwendig.

3 Vgl. §52a Abs. 2 S. 4 ERegG. 
Dies begründet sich einerseits in dem umfassenden Zusammenspiel diverser Strecken, verteilt auf den Personen- und Güterverkehr. So dürfte die im Einzelnen voraussichtlich zu meisternde Abstimmung von Verkehrsleistungen des SPFV, SPNV und SGV bei einer umfassenderen Betrachtung und deutlich größeren Netzabschnitten erschwert sein. Rechtliche Vorgaben für die Vorkonstruktion von Trassen erscheinen daher notwendig. Andererseits verbleibt auch bei der Ausgestaltung der Kapazitätsnutzungspläne hinsichtlich des freien Marktzugangs ein Spielraum für den SPFV, der zu Konflikten führen kann. Es stellt sich z. B. die Frage, wie der Infrastrukturbetreiber damit umgehen sollte, wenn SPFV-Unternehmen andere, als die im Plan vordefinierten Trassen anmelden. Um potenzielle Konflikte auszuschließen, müssten mindestens rechtliche Anpassungen der Vorrangkriterien erfolgen.

Sollte in Zukunft das Vergabesystem durch ein über die Erprobungsklausel erarbeitetes Modell mit Kapazitätsnutzungskonzept sowie Kapazitätsnutzungsplan angepasst werden, dürften darüber hinaus die folgenden drei rechtlichen Anpassungen notwendig sein:

\section{Definition von Systemtrassen}

Bisher sieht das deutsche Eisenbahnrecht im Allgemeinen keine Vorkonstruktion von Trassen vor. ${ }^{4}$ Dabei könnte eine entsprechende Definition Rechtsverbindlichkeit für die Deutschlandtakt-Trassen schaffen. Diese entsprechen in etwa bereits Systemtrassen, d. h. Fahrplantrassen, die der Betreiber der Schienenwege vorab und typischerweise abhängig von Erfahrungen mit dem Bedarf der Verkehrsarten konstruiert. Die anzumeldenden Trassen könnten über eine solche Definition im Eisenbahnregulierungsrecht bzw. einer zusätzlichen Anlage eingebettet werden. Alternativ könnte eine gesetzliche Vorschrift vorgeben, dass der Betreiber der Schienenwege entsprechende Systemtrassen in die Schienennetz-Benutzungsbedingungen aufzunehmen hat. Ein Vorteil davon wäre die jährliche Anpassungsmöglichkeit der wünschenswerten Trassen, beispielsweise aufgrund einer Evaluierung und Berücksichtigung der Nachfrageentwicklung.

\section{Anpassung des Trassenanmeldungsverfahrens}

Es müsste eine Vorrangregelung für Deutschlandtakt-Trassen geschaffen werden. Auf diese Weise könnten Konflikte, die über freie Trassenanmeldungen unabhängig vom Deutschlandtakt entstehen, verhindert werden. Deutschlandtakt-Trassen könnten z.B. eine gesonderte Berück-

4 Vordefinitionen von Trassen gibt es nur im Güterverkehr über das EURecht. Ähnliche Vorschriften zu Systemtrassen oder Anschlussbeziehungen für den SPV gibt es nicht (Durchführungsverordnung (EU) 913/2010). sichtigung im Eisenbahnregulierungsrecht erhalten. Dies könnte in Anlehnung an die bestehenden Regelungen zu besonderen Schienenwegen in § 57 ERegG erfolgen.

\section{Anreizmechanismen für Deutschlandtakt-Trassen im SPFV}

Auch mit einer Vorrangregelung der DeutschlandtaktTrassen sind Eisenbahnverkehrsunternehmen nicht gezwungen, diese Trassen anzubieten. Im schlimmsten Fall könnten SPFV-Unternehmen nur einzelne Abschnitte bedienen, wodurch sich das mit dem Deutschlandtakt angestrebte Verkehrsnetz durch die fehlende Einbindung des Fernverkehrs nicht abbilden würde. Für einen flächendeckenden Deutschlandtakt insbesondere im SPFV könnten zusätzliche Anreize zur Anmeldung von Trassen im Modell des offenen Marktzugangs (Open Access) gesetzt werden. Einerseits sollten diese Anreizmechanismen es attraktiver machen, die mit dem Deutschlandtakt gewünschten Trassen in ihrer vollen Länge anzumelden. Andererseits könnten zusätzliche Maßnahmen, wie beispielsweise geringere Trassenentgelte oder eine Subvention für die Anmeldung, weniger attraktiver Trassenangebote notwendig sein. Als potenzielle Möglichkeiten kämen daher entweder eine Anpassung des regulatorischen Rahmens zur Entgeltbildung infrage oder eine direkte Förderung bei der Anmeldung spezifischer Trassen des Zielfahrplans. Ein Beispiel hierfür ist die Trassenpreissenkung im SGV. Diese trat 2018 mit der Richtlinie zur Förderung des SGV über eine anteilige Finanzierung der genehmigten Trassenentgelte (af-TP) in Kraft. Im Speziellen ist eine Einschränkung der Förderung auf solche Trassen anzustreben, die von den SPFV-Eisenbahnverkehrsunternehmen nicht bereits ohne Förderung angemeldet würden. Auf diese Weise würde der Umfang der Subventionen reduziert, da ein Großteil der Trassen gemäß ihrer Vorkonstruktion für den Zielfahrplan bereits eigenwirtschaftlich zu erbringen sein sollte.

\section{Umgestaltung des SPNV empfehlenswert}

Eine Alternative zu den rechtlichen Anpassungen ist die grundsätzliche Änderung des Marktmodells im SPFV. Ein Wettbewerb „um den Markt”, ähnlich dem SPNV, könnte die aufgezeigte Problematik konfligierender Streckenanmeldungen und einer fehlenden Umsetzung der Deutschlandtakt-Trassen verhindern. Der Wechsel zu einem Ausschreibungswettbewerb könnte einen eigenen Bundesaufgabenträger vorsehen, der die für den Fernverkehr im Deutschlandtakt vorgesehenen Strecken ausschreibt. Alternativ könnte auch ein Wechsel zu einem Konzessionswettbewerb erfolgen, wie er z. B. in der Schweiz existiert. Beide Möglichkeiten bieten den Vorteil, dass durch den organisatorischen Wandel eine Sicherstellung der angestrebten SPFV-Verkehrsleistungen ge- 
schaffen werden könnte. Ein weiterer Vorteil ist, dass mit der Vergabe von Strecken über einen längeren Zeitraum die Zugangssicherheit im SPFV erhöht werden könnte. Diese wird insbesondere von Wettbewerbern im SPFV als Markteintrittsbarriere kritisiert.

Abhängig von der Ausgestaltung ergeben sich mit dem angepassten Marktmodell gegebenenfalls auch Nachteile. Beispielsweise würde im Vergleich zu dem gegenwärtigen Open-Access-Modell die Zahl der Wettbewerbsparameter sinken. Eine höhere Zahl an Wettbewerbsparametern bietet zwar keine Sicherheit für einen stärkeren Wettbewerb. Jedoch geht bei einem freien Marktzugang durch die Möglichkeit des Markteintritts potenzieller Wettbewerber ein gewisser Druck aus, der sich positiv auf die Qualität und Effizienz des Systems auswirken kann. Bei einem Wettbewerb „um den Markt“ ist demgemäß durch die einhergehende regionale Monopolisierung ein potenzieller Qualitätsverlust zu befürchten. Diesen Nachteilen könnte allerdings mit der Vorgabe von Effizienz- und Qualitätskriterien bzw. entsprechenden Anreizelementen vorgebeugt werden.

Eine Kritik an dem Wechsel zu einem Wettbewerb „um den Markt" ist der Umstand, dass der Fernverkehr prinzipiell eigenwirtschaftlich zu erbringen sei und der Marktmodellwechsel mit Subventionen für Strecken bzw. Streckenbündel einhergehen werde. $\mathrm{Zu}$ berücksichtigen ist daher, dass mit einer gebündelten Vergabe von besonders wirtschaftlich und weniger wirtschaftlich zu bedienenden Strecken die Notwendigkeit einer Subventionierung unrentabler Strecken vermindert würde. Des Weiteren ist auch ohne eine Anpassung des Marktmodells für die Umsetzung möglichst vieler Trassen des Deutschlandtakts eine finanzielle Anreizsetzung im SPFV notwendig.

Im Fall eines zukünftigen Ausschreibungswettbewerbs oder Konzessionsmodells im SPFV sollte dieser unbedingt wettbewerblich ausgestaltet werden. Die Monopolkommission rät dazu, einen neutralen Bundesaufgabenträger zu bestimmen (Monopolkommission, 2021). So würde eine wettbewerbliche Vergabe der Strecken bzw. Streckenbündel sichergestellt. Zudem sollten Vergabeanforderungen an Effizienz- und Qualitätskriterien geknüpft werden. Auf diese Weise könnten mögliche Wettbewerbsverzerrungen und Effizienzeinschränkungen durch die beschränkte Bedienung (durch ein Unternehmen) verhindert werden. Auch könnte mit der Vergabe von Streckenbündeln verhindert werden, dass für die Erbringung einzelner, weniger wirtschaftlicher Strecken höhere Subventionen gezahlt werden müssten. Weiterhin könnte verhindert werden, dass neue Marktteilnehmende nur wenig wirtschaftliche Strecken in dem Vergabe- oder Konzessionsmodell erhalten würden. Mit der Umgestaltung des SPFV könnte langfristig die Umsetzung des Deutschlandtakts erreicht werden.

\section{Ausblick}

Damit im Rahmen des Deutschlandtakts tatsächlich mehr Verkehr auf die Schiene verlagert werden kann, ist es wichtig, dass dieser für Eisenbahnverkehrsunternehmen und Reisende attraktiv ausgestaltet wird. Eine wettbewerbliche Umsetzung trägt dazu grundsätzlich bei. $\mathrm{Zu}$ empfehlen ist die Anpassung des Marktmodells im SPFV hin zu einem Ausschreibungs-/Konzessionsmodell. Durch diese Änderung könnte eine umfängliche Einführung der Deutschlandtakt-Trassen erfolgen. Gleichzeitig könnte sich durch die Umstellung auch im Fernverkehr mehr Konkurrenz einstellen. Voraussetzung für diese Ausgestaltung des Bahnmarkts und die erwartete Verkehrssteigerung ist allerdings eine umfassende Anpassung der Infrastruktur. Diese erfordert in den kommenden Jahren und Jahrzehnten zusätzliche Investitionen in das Schienennetz sowie weitere regulatorische Anpassungen für den Schienennetzbetreiber, z. B. zur Steigerung der für den Taktfahrplan notwendigen Pünktlichkeit.

\section{Literatur}

BMVI (2020a), Zielfahrplan Deutschlandtakt, dritter Gutachterentwurf, Juni 2020, Akteurskonferenz, 15. Juli.

BMVI (2020b), Zukunftsbündnis Schiene, Abschlussbericht der Arbeitsgruppen des Zukunftsbündnis Schiene, Mai.

BMVI (2021), Abschlussbericht zum Zielfahrplan Deutschlandtakt. Grundlagen, Konzeptionierung und wirtschaftliche Bewertung, 31. August.

Böttger, C. (2020), Eine kritische Würdigung des Masterplans Schiene, Bahn-Report, 5/2020, 8 .

Monopolkommission (2019), 7. Sektorgutachten Bahn (2019): Mehr Qualität und Wettbewerb auf die Schiene, Tz. $60 \mathrm{ff}$.

Monopolkommission (2021), 8. Sektorgutachten Bahn (2021): Wettbewerb in den Takt!, Tz. $30 \mathrm{f}$.

Title: Competition in the "Deutschlandtakt"

Abstract: The new federal government has retained the transport policy goal of increasing rail traffic. In its 24 November 2021 agreement, the coalition confirmed further investments in rail infrastructure. In addition, a national integrated timetable, the "Deutschlandtakt", has been created to achieve this goal. In order to increase rail traffic, the Deutschlandtakt must be competitively implemented. The authors discuss possible regulatory adjustments and their impact on the rail market. A suitable instrument is the redesign of longdistance passenger rail transport towards a tender or concession model. The redesign can contribute to increased competition, a more attractive rail market as well as an increase in rail traffic. 